Meta

Journal des traducteurs

Translators' Journal

\title{
Télévision : Les artefacts de la traduction-adaptation
} Le cas de la sitcom

\section{Yannicke Lebtahi}

Volume 49, numéro 2, juin 2004

URI : https://id.erudit.org/iderudit/009366ar

DOI : https://doi.org/10.7202/009366ar

Aller au sommaire du numéro

\section{Éditeur(s)}

Les Presses de l'Université de Montréal

\section{ISSN}

0026-0452 (imprimé)

1492-1421 (numérique)

Découvrir la revue

Citer cette note

Lebtahi, Y. (2004). Télévision : Les artefacts de la traduction-adaptation : le cas de la sitcom. Meta, 49(2), 401-409. https://doi.org/10.7202/009366ar
Résumé de l'article

L'étude comparative des dialogues entre les versions originales (V.O.), les versions originales sous-titrées en français (V.O.S.T.) et les versions françaises doublées (V.F.) d'un numéro de trois sitcoms différentes : Dingue de toi, Friends et Les dessous de Véronica, permet d'observer quatre catégories d'artefacts de traduction-adaptation :

1. les écarts de traduction dus à l'adaptation des références culturelles ;

2. les infidélités de traduction qui transforment la personnalité des protagonistes;

3. les modifications des dialogues qui affectent l'équilibre originel entre la médiation verbale et la médiation visuelle ;

4. les libertés de traduction qui peuvent altérer la trame narrative. Les résultats détaillés sont présentés dans l'analyse de la sitcom Dingue de toi. La comparaison de la V.O.S.T. et de la V.F. montre que la V.O.S.T. est moins infidèle que la V.F. Reste que la traduction-adaptation est délibérément conçue comme une opportunité de repositionnement pour la V.O.S.T. et de changement de cible pour la V. F., avec l'hypothèse d'une demande différente du public français. Toutefois, les altérations de la trame narrative et les incohérences de reciblage montrent que la traduction-adaptation souffre d'une insuffisance des moyens consacrés au travail de la post-production.
Tous droits réservés (C Les Presses de l'Université de Montréal, 2004
Ce document est protégé par la loi sur le droit d'auteur. L'utilisation des services d'Érudit (y compris la reproduction) est assujettie à sa politique d'utilisation que vous pouvez consulter en ligne.

https://apropos.erudit.org/fr/usagers/politique-dutilisation/ 


\title{
BLOC-NOTES
}

\section{Télévision: Les artefacts de la traduction-adaptation. Le cas de la sitcom}

\author{
RÉSUMÉ \\ L'étude comparative des dialogues entre les versions originales (V.O.), les versions originales sous-titrées en \\ français (V.O.S.T.) et les versions françaises doublées (V.F.) d'un numéro de trois sitcoms différentes: Dingue \\ de toi, Friends et Les dessous de Véronica, permet d'observer quatre catégories d'artefacts de traduction- \\ adaptation: \\ 1) les écarts de traduction dus à l'adaptation des références culturelles; \\ 2) les infidélités de traduction qui transforment la personnalité des protagonistes; \\ 3) les modifications des dialogues qui affectent l'équilibre originel entre la médiation verbale et la médiation \\ visuelle; \\ 4) les libertés de traduction qui peuvent altérer la trame narrative. \\ Les résultats détaillés sont présentés dans l'analyse de la sitcom Dingue de toi. \\ La comparaison de la V.O.S.T. et de la V.F. montre que la V.O.S.T. est moins infidèle que la V.F. Reste que \\ la traduction-adaptation est délibérément conçue comme une opportunité de repositionnement pour la \\ V.O.S.T. et de changement de cible pour la V. F., avec l'hypothèse d'une demande différente du public fran- \\ çais. Toutefois, les altérations de la trame narrative et les incohérences de reciblage montrent que la traduc- \\ tion-adaptation souffre d'une insuffisance des moyens consacrés au travail de la post-production.
}

\section{ABSTRACT}

The comparative study of dialogues between the original versions, the original versions with French subtitles and the French versions of three sitcoms: Friends, Mad About You and Veronica's closet demonstrates four categories of translation-adaptation's artefact:

1) the translation's gaps due to cultural adaptation;

2) the translation's inaccuracies that change the characters' personality;

3) the dialogues' modifications that affect the initial equilibrium between verbal mediation and visual mediation;

4) the translation's liberties that can corrupt the narrative texture.

The detailed results are presented in analysing the sitcom Mad about you.

The comparison between the original version with French subtitles and the French version shows that the former is more faithful than the latter. Moreover translation-adaptation is deliberately seen as an opportunity of repositioning for the original version with French subtitles and as a change of target for the French version, by virtue of an hypothesis of a difference in the French audience request. However, the narrative texture corruption and the incoherence of the target's change show that translation-adaptation suffers from an insufficiency of resources invested in the post-production work.

\section{MOTS-CLÉS/KEYWORDS}

adaptation, dialogues, écarts de traduction, infidélités de traduction, sitcom

Notre propos est d'exposer les résultats d'une recherche sur les artefacts de la traduction-adaptation de la version originale vers la version originale sous-titrée en français et vers la version française doublée dans le cas de la sitcom.

Version anglo-saxonne légère de la comédie de situation, la sitcom mérite, en tant qu'objet d'analyse, une attention particulière car c'est un genre télévisuel contemporain en constante évolution.

La sitcom est exemplaire de l'économie de la télévision et des enjeux qui lui sont attachés, notamment d'un point de vue culturel. C'est une production sérielle, caractérisée par la rapidité de la réalisation et de la stricte modestie des moyens de production. La sitcom est conçue comme un produit exportable dans le cadre d'un espace télévisuel élargi et globalisé.

La sitcom se distingue de la série télévisuelle classique par diverses caractéristiques. Tout d'abord, elle n'excède généralement pas 30 minutes. Elle est découpée en saisons. Elle est enregistrée en studio avec une équipe restreinte. Les acteurs évoluent toujours dans les mêmes décors. Leur jeu de scène est exagéré. Les personnages ont des traits de caractère marqués, les acteurs n'ayant qu'une faible marge de liberté pour l'interprétation.

Inspirée des dramatiques radiophoniques apparues dans les années 1940, la sitcom constitue une version télévisuelle du spectacle vivant car elle est enregistrée sur scène devant un vrai public ou simulé 
comme tel avec des rires en boîte (Jost 1999). Le travail de la bande-son y fait donc l'objet d'un investissement plus conséquent que dans les autres types de fiction télévisuelle sérielle. Par ailleurs, si les conditions de tournage sont des plus sommaires, «c'est que l'anecdote passe plus par la médiation verbale que par la médiation télévisuelle et que quelques décors suffisent pour suivre ces récits de paroles» (Jost 1999: 114).

Compte tenu de la prééminence de la médiation verbale dans la sitcom, nous pouvons nous interroger sur sa réception par le téléspectateur selon les différentes modalités de diffusion: version originale, version française et version sous-titrée.

En d'autres termes, le rôle de la traduction-adaptation de la sitcom dans la construction du sens par le téléspectateur nous paraît être une question essentielle.

Est-ce que la traduction et l'adaptation modifient ou non le sens de la narration et donc l'identité de la sitcom? Si tel est le cas, quels sont les changements qui s'opèrent et pourquoi?

\section{Corpus et méthode}

Le corpus de notre recherche se compose des différentes versions, originale (V.O.), originale sous-titrée en français (V.O.S.T.) et française doublée (V.F.) d'un numéro de trois sitcoms différentes : Dingue de toi, Friends et Les dessous de Véronica.

Nous avons mené une étude comparative des dialogues entre les différentes versions, originales, françaises et sous-titrées. Pour l'interprétation de nos observations, nous avons tenu compte des problèmes posés pour la traduction. En effet, le passage à la version française doit intégrer les mouvements des lèvres des interprètes et la durée des dialogues. Pour la version sous-titrée, le temps de lecture du téléspectateur induit lui aussi des contraintes.

Les résultats obtenus pour chacune des sitcoms étudiées ont été ensuite confrontés. Pour favoriser la lisibilité de l'exposé de notre recherche, nous présenterons ici notre analyse de la sitcom Dingue de toi. Ces résultats sont corroborés par les résultats issus de l'analyse des deux autres sitcoms.

\section{Présentation de la sitcom Dingue de toi (Mad About You)}

Dingue de toi a été créée en 1992 par Paul Reiser, l'interprète d'un des protagonistes. Les sept saisons qui se sont déroulées à l'écran jusqu'en 1999 témoignent de l'immense succès qu'elle a connu aux États-Unis.

Aux États-Unis, la diffusion en prime time - 20h00 - vise un public large et non ciblé. En France, la programmation est légèrement différente, cette sitcom y étant moins célèbre que Friends par exemple. Sur TFI, chaîne généraliste, leader en part de marché, Dingue de toi est diffusée à 16h30 le samedi après-midi. L'audience potentielle est plus réduite, mais la programmation vise également un public familial. Depuis le 6 octobre 1999, Dingue de toi est diffusée sur Comédie, chaîne câblée, à 20h30 en version française (V.F.) et à 0 h45 en version originale sous-titrée (V.O.S.T.). Le positionnement de la chaîne Comédie repose sur le rire et le divertissement que procurent aisément les sitcoms. Le fait que la chaîne procède à une double programmation, V.F. et V.O.S.T., montre qu'elle segmente son audience, en visant un public familial en début de soirée avec la V.F. et un public jeune, averti et puriste, le soir très tard avec la V.O.S.T.

Dingue de toi met en scène un jeune couple marié depuis 5 mois. Paul (Paul Reiser) et Jamie Buchman (Helen Hunt) nous ouvrent les portes de leur toute nouvelle vie à deux, une vie bien mouvementée. Tous deux jonglent entre leur carrière, leur belle-famille respective, leurs amis, leur chien, la jungle de New York et tentent malgré tout de fixer les règles de leur vie de couple. Pour Jamie et Paul, le mariage était une évidence: ils étaient faits l'un pour l'autre. Mais la lune de miel terminée, leurs caractères très différents doivent s'apprivoiser et là... c'est une autre histoire! Grandes discussions et batailles de mots en perspective! Helen Hunt a reçu, en février 1997, deux Golden Globe Awards pour son rôle de jeune femme moderne, intelligente et drôle dans cette comédie délicieuse.

Le numéro analysé s'intitule "Jamie a un secret» ("The wedding affair»). Nous pouvons d'ors et déjà constater un écart de traduction-adaptation dans ce titre. Il aurait pu se traduire par «l'incident nuptial» ou par «l'incident conjugal». Mais le terme «wedding» détient ici ce double sens car, nous le verrons dans l'analyse, c'est un double incident, nuptial et conjugal, qui constitue la trame narrative du numéro présenté. Paul et Jamie se rendent à un mariage. Paul a dévoilé au père de la mariée un secret de celle-ci: elle a eu une aventure au début de leur relation. Il apprend alors que Jamie, sa propre femme, lui dissimule également un secret. Il en découle que Jamie est contrainte de le lui révéler. 


\section{Analyse}

De l'analyse des traductions, V.F. et V.O.S.T., de ce numéro, nous avons déduit quatre catégories d'items:

1) les écarts de traduction dus à l'adaptation des références culturelles;

2) les infidélités de traduction qui transforment la personnalité des protagonistes;

3) les modifications des dialogues qui affectent l'équilibre originel entre la médiation verbale et la médiation visuelle;

4) les libertés de traduction qui peuvent altérer la trame narrative.

Chacune de ces catégories constitue une heuristique particulière des artefacts de la traductionadaptation d'une marchandise culturelle: la sitcom. Il s'agit non pas de constater des types formels d'opération d'un point de vue purement linguistique mais de qualifier des artefacts issus d'une post-production à destination d'un nouveau marché.

Nous présenterons ces catégories d'artefacts l'une après l'autre. Pour chacune de ces catégories, nous exposerons les observations empiriques ou items qui les déterminent.

\section{1) Les écarts de traduction dus à l'adaptation des références culturelles}

Le traducteur, que ce soit en V.F. ou en V.O.S.T., doit faire un effort d'adaptation des références culturelles afin que ces versions puissent être reçues par les téléspectateurs français. Pour cela, il modifie le texte original en lui restant plus ou moins fidèle. Alors qu'il dispose d'une grande liberté avec la V.F., le traducteur est contraint d'être plus fidèle pour la V.O.S.T. car le téléspectateur, s'il comprend l'anglais, est en mesure de comparer le texte original et la traduction.

Dans la première séquence:

- En V.O., Jamie fait référence au fait qu’elle a aidé la mariée à maigrir: «I talked her to Weight Watchers.»

- En V.O.S.T., le dialogue conserve le même sens, en évitant cependant la traduction littérale: «Je l'ai encouragée à maigrir.» La traduction fait l'impasse sur la référence à Weight Watchers, car elle n'aurait pas le même pouvoir d'évocation dans le contexte français. Cette méthode de régime est moins connue et moins répandue en France qu'aux États-Unis.

- En revanche, en V.F., l'idée de perte de poids est évacuée au profit d'un autre inconvénient esthétique: «Dire que je l'ai aidée à soigner son acné!»

Alors que la V.O.S.T. reste assez fidèle à la V.O., l'inconvénient évoqué dans la V.F. est beaucoup plus ciblé que dans la V.O. En termes de concept de soi et d'identification, il concerne plus une cible jeune.

Toujours dans la première séquence:

- En V.O., Paul ne veut pas donner la machine à confectionner des pâtes. Cette prérogative lui permet de se sentir italien. Jamie lui fait alors remarquer qu'il aime se sentir italien: "You like feeling Italian.»

- En V.O.S.T., le traducteur reste littéralement fidèle: «Tu aimes te sentir italien.»

- Cependant en V.F., le traducteur a remplacé la référence à l'italienneté par une référence à une chanson française à succès. Paul dit qu'il se sent «presque gai comme un Italien»; ce à quoi Jamie répond: "Quand il y a de l'amour et du vin.»

Dans la V.F., le traducteur a supposé que l'italienneté n'était évocable que dans le cadre du meltingpot américain et surtout pas dans le contexte d'intégration à la française des immigrés, où les caractéristiques communautaires d'origine sont subordonnées à la nouvelle culture d'adoption. En voulant éviter le risque d'incompréhension d'une référence au contexte culturel américain, le traducteur a substitué une référence culturelle, en l'occurrence une chanson interprétée par Nicole Croisille, qui n'est pas forcément partagée par le plus grand nombre. Nous pouvons nous interroger sur la pertinence d'une telle référence, qui dénote d'une orientation sur un public populaire plus ciblé, connaissant la chanson évoquée.

Dans la troisième séquence:

- Le dialogue de la V.O. fait référence à un jeu vidéo américain dans la réplique d’Ira, «Smart bomb sparky!»

- En V.O.S.T., la traduction évite cette référence pour néanmoins garder la notion de lumière (spark = étincelle) : «T’es vraiment une lumière!»

- Dans la V.F., le traducteur a préféré une formule familière qui ne conserve ni la référence américaine au jeu vidéo ni la référence littérale au mot «spark»: «Finement joué mon grand!» 
La V.O.S.T. prend en compte la contrainte sémantique de la V.O. en évitant cependant la référence culturelle au jeu vidéo. Paradoxalement, il se produit, dans la V.F., l'opération opposée par rapport à un item précédent. Alors que la réplique initiale s'ancre dans la culture des jeunes, avec la référence à un jeu vidéo, sa traduction vise à être comprise par le plus grand nombre.

Dans la cinquième séquence:

- En V.O., Paul demande à Jamie quel est son secret, a-t-elle a acheté un chapeau? Jamie répond: «Who am I? Lucy?» La réplique renvoie à une autre sitcom intitulée I love Lucy, où il doit être question de chapeau. Ici, Dingue de toi met en avant son statut télévisuel en sollicitant la culture téléphilique du téléspectateur (Jost 1999).

- En V.O.S.T., la référence est explicitée, au risque de perdre le trait d'humour car il n'y a plus le clin d'œil complice avec le public: «Tu te crois dans I love lucy?» La série n'étant pas connue en France, le traducteur est contraint à une explicitation de la référence.

- En revanche dans la V.F., totalement libéré des contraintes du texte original, le traducteur a gardé l'humour de la réplique en occultant toutefois la référence culturelle télévisuelle et américaine: «J'ai acheté un chapeau... J'ai acheté un chapeau de Catherinette.»

Le traducteur fait référence, dans la V.F., à la tradition populaire française, une Catherinette étant une jeune vierge, à l'honneur à la Sainte-Catherine, le 25 novembre. La complicité avec le public sort cependant de l'univers télévisuel pour épouser celui des traditions populaires.

Au travers de ces items de traduction d'éléments de dialogue reposant sur des références culturelles, nous pouvons observer des écarts notables.

Dans la traduction en V.O.S.T., le traducteur tient compte des contraintes sémantiques, il reste relativement fidèle au texte mais dans la moitié des cas, il gomme la référence culturelle. Que certaines de ces références soient conservées s'explique certainement par le fait que le public d'une V.O.S.T. est motivé par l'authenticité par rapport à la version originale.

Dans la traduction en V.F., le traducteur s'affranchit totalement des contraintes sémantiques, soit la traduction n'a rien à voir avec le texte original, soit elle est considérablement adaptée. Plus grave, ces glissements induisent une modification dans le type de public visé. Nous pourrions comprendre une telle modification si elle s'opérait de façon homogène mais elle apparaît équivoque. En effet, par deux fois, elle cible un public plus populaire. Pour une occurrence, elle vise la compréhension par un public plus large, tandis que pour la première occurrence précitée, le public visé semble plus jeune, avec la référence à l'acné juvénile. Il y a donc une contradiction qui dénote un manque de maîtrise du procédé.

\section{2) Les infidélités de la traduction qui transforment la personnalité des protagonistes}

En s'éloignant du texte d'origine, le traducteur peut aussi transformer les traits de personnalité des protagonistes et donc l'identité de la sitcom.

Dans la deuxième séquence:

Paul est le seul en smoking à la réception. À qui la faute?

- En V.O., Paul dit qu'il a fait le mauvais choix: «I think I opted wrong.» Donc, Paul est responsable de son erreur. Il est un peu niais.

- En V.O.S.T., la traduction est fidèle: «Je crois que j'ai fait le mauvais choix.»

- En V.F., il rejette la faute sur Jamie: «Oui, ben, je crois que tu n’as pas bien lu.» Implicitement le récit est modifié, Jamie a spécifié à son mari qu'il devait revêtir un smoking. D’ailleurs sa réplique ne laisse aucun doute: "C'était pourtant noté sur les invitations.»

Le traducteur sous-entend, dans la V.F., que Jamie gère le couple. La mésaventure de Paul est imputable à la domination de sa femme.

Encore dans la deuxième séquence:

Jamie fait remarquer à Paul que c'est son cousin Ira qui joue dans l'orchestre pour le mariage.

- Dans la V.O., c'est Paul qui a fait engagé son cousin: «I can't believe you got hired your cousin Ira!»

- Au contraire, en V.O.S.T., ce n’est pas Paul qui l’a fait engagé: «Je n’arrive pas à croire qu'ils ont engagé ton cousin Ira!»

- La V.F., quant à elle, respecte le sens original: «Je ne peux pas croire que tu aies fait engager ton cousin Ira!»

Il y a manifestement une erreur de traduction. La V.O.S.T. s'écarte de la V.O. En V.O., Paul est un homme qui aide sa famille et en V.O.S.T., il n'y a plus cette idée. Nous ne percevons donc pas Paul comme 
quelqu'un qui a un sens aigu de la famille. C'est un trait de sa personnalité qui disparaît. Ce qui étonne par rapport aux autres items, c'est que la V.F. respecte plus le sens de la V.O. que la V.O.S.T. Nous pouvons supposer qu'il s'est produit une erreur involontaire du traducteur.

Toujours dans la deuxième séquence:

Jamie annonce à Paul qu'il vient de commettre un impair en révélant le secret de la mariée.

- En V.O., il demande à Jamie si elle plaisante: «Oh, get out over here!»

- La même idée est reprise en V.O.S.T.: «Arrête ton char!»

- En revanche, en V.F., Paul ne fait pas de phrase complète: «Qu'est-ce-que...»

Dans la V.F., le caractère et la personnalité de Paul ne sont pas aussi affirmés que dans la V.O. et dans la V.O.S.T. En France, il passe plus pour quelqu'un d'un peu mou, qui a du mal à s'exprimer, alors qu'à l'origine, il a une personnalité assez forte.

Dans la quatrième séquence:

Paul et Jamie dansent pour mettre de l'ambiance. Jamie laisse Paul seul un moment et quand il la rejoint, il s'étonne qu'à présent tout le monde danse.

- En V.O., Jamie lui dit que c'est normal parce qu'eux ne dansent plus: «Sure, we're gone.»

- En V.O.S.T., le traducteur est resté fidèle à la réplique d'origine: «Normal, on a arrêté.»

- En V.F., Jamie est plus sarcastique avec Paul: «Bah oui, t'es parti.»

Dans la V.F., Paul est plus niais, moins proche de sa femme que dans la V.O. et, dans la V.O.S.T., Jamie est agressive. La V.F. positionne le couple comme moins complice, moins soudé.

Dans la cinquième séquence:

- Dans la V.O., Jamie arrive à table et, voyant les assiettes vides, demande s'ils ont raté le repas: «Did we miss food?» Il y a un trait d'humour dû à la situation car nous savons depuis le début qu'elle a faim.

- La V.O.S.T. renvoie au même sens, «On a loupé la bouffe», avec une connotation plus argotique. En V.O., il n'y a pas l'idée de «bouffe» mais de nourriture. Le traducteur prête à Jamie un vocabulaire qu'elle n'a pas.

- En V.F., Jamie a le côté plus affamé de quelqu’un qui ne vient au mariage que pour manger: «Y’a rien à manger?»

La V.O.S.T. donne une image assez vulgaire de Jamie qui n'est pas fidèle à la V.O. Dans la V.F., Jamie semble plus sans gêne que dans la V.O. L’humour repose sur ce côté sans gêne.

Toujours dans la cinquième séquence:

Paul insiste pour savoir quel est le secret de Jamie et lui dit que si elle le lui avoue, il lui montrera à quel point il l'aime.

- En V.O., Jamie dit qu'elle l'aime aussi et qu'il vaut mieux en rester là : "I love you too, so let's not do this.»

- La V.O.S.T. respecte tout à fait la V.O.: «Je t'aime aussi, restons-en là !» L'intégrité du caractère de Jamie est conservée.

- En V.F., en revanche, elle est une nouvelle fois plus autoritaire: «Moi aussi je t'aime, alors tu arrêtes tout de suite!»

Dans la V.F., le traducteur prête à Jamie un ton agacé qui n'existe pas en V.O. et lui crée un tempérament de femme dominatrice.

Dans la sixième séquence:

Jamie avoue à Paul qu'elle a eu une aventure avec quelqu'un d'autre et que cela lui a permis de l'aimer deux fois plus.

- En V.O., Paul répond ironiquement qu'il va envoyer à cet homme une carte: «Thank you for ironing my wife's things.» ce qui, traduit littéralement, donne: «Merci d'avoir repassé les petites affaires de ma femme» et peut signifier par extension: «Merci de t'être occupé des petites affaires de ma femme.»

- En V.O.S.T., la traduction est assez vulgaire: «Merci d'avoir tringlé ma femme!» Le traducteur a sans doute voulu garder l'image du triangle ou de la tringle que l'on a avec le repassage des vêtements en V.O. (to iron = repasser), mais cela ne relève pas du même registre de langue. À la limite, pour être trivial, il aurait pu dire: «Merci d'avoir repassé les petites culottes de ma femme.»

- En V.F., la traduction opère un glissement. De ce qui s'est passé entre Jamie et son ex-amant, nous passons aux conséquences pour Paul: «Merci de m’avoir laissé ma femme!» 
Au travers de la V.O.S.T., Paul apparaît comme un personnage extrêmement vulgaire. Le discours de Paul dans la V.F. relève quelque peu du machisme; c'est à l'initiative de cet amant que Paul doit sa relation actuelle avec Jamie. Cela laisse supposer que ce n'est pas Jamie qui a pris l'initiative de la rupture. Nous retrouvons à nouveau l'idée d'un couple moins soudé, dont l'existence est plus soumise au destin qu'à la volonté des personnages.

Ces différentes occurrences montrent que la traduction dans les deux versions, V.O.S.T. et V.F., modifie de façon substantielle la personnalité des protagonistes. Dans la V.O.S.T., Paul et Jamie emploient un langage vulgaire. Dans la V.F., Paul devient une personne moins fine et perd, par rapport à la V.O., plusieurs occasions d'exprimer ses qualités. Il passe alors au second plan et laisse, à son détriment, sa femme prendre le dessus. Toujours dans la V.F., Jamie est plus forte, plus affirmée, voire plus autoritaire. Le traducteur n'a pas respecté l'équilibre du couple. La sitcom de Paul Reiser raconte les aventures d'un couple marié. Elle vante les mérites, à la fois de Jamie et de Paul. Quoi qu'il arrive, l'un soutiendra l'autre. Dans ce numéro, Jamie répare les pots cassés lorsque Paul commet un impair et pardonne à sa femme une erreur de jeunesse. Ils sont adultes car mariés. La V.F. brise cet équilibre, ce qui change l'interprétation de la série et les opportunités d'identification du public aux protagonistes. Alors que les Américains seront davantage enclins à observer un couple modèle dans des situations de la vie qui prêtent à sourire, les Français y verront plutôt les aventures d'un couple moins soudé conduit à surmonter des difficultés. Cette modification induit un changement d'identité de la sitcom et semble donc délibérée.

\section{3) Les modifications dans les dialogues qui affectent l'équilibre originel entre la médiation verbale et la médiation visuelle}

Lors du passage de la V.O. à la V.F., la traduction peut être le prétexte à une modification du rapport entre la médiation verbale et la médiation visuelle. Cette modification ne se produit pas dans la V.O.S.T., puisque l'opération de sous-titrage reste subordonnée au dialogue original.

Dans l'introduction:

Jamie dit à Paul qu'elle ne va pas l'attendre. Il répond que si.

- Dans la V.O., elle réplique alors: «You wanna bet?» Ce qui signifie: «On parie.» À ce même moment, elle lui présente son dos pour qu'il remonte la fermeture éclair de sa robe.

- En V.F., la réplique disparaît. Il n'y a pas de suite à ce que disait Paul mais les paroles de Jamie sont axées sur la situation. Elle dit: "Tu veux m'aider?»

Le dialogue de la V.F. n'a plus rien à voir avec le dialogue original. Il s'agit juste de meubler le mouvement des lèvres de Jamie en soulignant les gestes des personnages.

Il en est de même quelques instants après, toujours dans l'introduction:

Paul dit à Jamie que cela ne sert à rien de se presser car ils sont en avance et qu'elle va l'ennuyer en insistant sans cesse.

- Dans la V.O., elle lui répond qu'elle en prend le risque: "I take the risk. » Au même moment, elle saisit la télécommande pour éteindre la télévision.

- En V.F., le traducteur n'a pas retenu sa réplique, mais l'a remplacée par un aparté qui souligne son geste avec la télécommande; "Tu sais quoi? Confisquée!»

Dans la V.F., le traducteur renforce l'insistance de Jamie, en subordonnant le verbal au visuel.

Dans la deuxième séquence:

Jamie s'avance vers le serveur pour prendre quelques gâteaux apéritif.

- Dans la V.O., à ce moment-là, elle fait signe à Paul de lui apporter une assiette sans que l'on entende sa voix, juste pour qu'il puisse le lire sur ses lèvres.

- En V.F., sans doute pour une meilleure compréhension du téléspectateur, le traducteur a ajouté des paroles: «Donne une assiette, merci!»

Nous retrouvons donc dans la V.F. la subordination du verbal au visuel avec ici la création de discours ex nihilo.

Toujours dans la deuxième séquence:

- En V.O., Jamie demande à Paul comment il trouve la robe de la mariée. Il répond qu'il l'aime, Jamie fait alors la grimace et ils vont ensuite saluer les mariés.

- En V.F., le traducteur a souligné la grimace en créant le dialogue à la suite de la réponse de Paul. Jamie répond: «Beurk!» Puis Paul: «Non.» Et enfin, Jamie, s'adressant aux mariés: «Bonjour!» 
Nous retrouvons dans la V.F., la création ex nihilo de discours subordonnés au jeu scénique, donc à la médiation visuelle.

Quelques instants après, toujours dans la deuxième séquence:

Paul vient de révéler le secret de la mariée au père de celle-ci.

- Dans la V.O., nous la voyons alors essayer de rassurer son père mais sans entendre ce qu'elle dit.

- En V.F., le traducteur crée la réplique de la mariée: «Non, non, non, non, tu peux me croire Papa.

C'est pas vrai. Je te jure. C'est pas vrai!»

Cette création ex nihilo vise à mieux nous faire suivre ce qui est au centre du récit de cet «incident nuptial». Ici, la médiation verbale, créée de toutes pièces, vise à renforcer à nouveau la médiation visuelle.

Dans la septième séquence:

Paul et Jamie vont s'en aller. Ils veulent dire bonsoir à d'autres invités.

- Dans la V.O., Jamie embrasse des amies.

- Dans la V.F., le traducteur donne la parole à Jamie et à une invitée. Jamie: «Bon, je t’appellerai.» L'invitée dit à Paul: «Allez, bonsoir Paul.»

Dans la V.F., un petit dialogue a sans doute été rajouté pour rendre cette séquence d'au revoir plus authentique. Nous retrouvons la redondance de la médiation visuelle par la création d'une médiation verbale liée.

Ces différents éléments montrent que le passage de la V.O. à la V.F. modifie délibérément l'équilibre entre la médiation verbale et la médiation visuelle. Nous observons ici un net renforcement de la médiation verbale par la création ex nihilo ou, dans une moindre mesure, la substitution de discours, subordonnés à la médiation visuelle. Il semble que le parti pris de la V.F. suppose que le public français a besoin d'explicitations d'ordre verbal.

\section{4) Les libertés de traduction qui peuvent altérer la trame narrative}

Dans les trois catégories précédentes, nous avons pu observer des écarts ou des modifications qui peuvent trouver une explication dans une politique délibérée d'adaptation au contexte culturel, à un nouveau positionnement de la sitcom ou à une autre forme de médiation. Nous avons choisi de regrouper dans une dernière catégorie les items où le traducteur prend des libertés avec le texte original sans que nous puissions avancer une explication satisfaisante. Nous avons ensuite observé que ces libertés pouvaient induire une modification injustifiable de la trame narrative.

Dans la quatrième séquence:

Un couple aborde Paul et Jamie.

- En V.O., ils échangent de simples «Bonjour», «Comment allez-vous?» qu'ils répètent plusieurs fois: «Hey there, how are you?», «Hi, how are you? Good to see you!» etc.

- En V.O.S.T., la traduction est fidèle.

- En V.F., le traducteur a remplacé les répétitions de la V.O. par des banalités qui peuvent s'échanger au cours de telles occasions : «Quelle belle soirée!», «Oh oui! C'est vrai qu'on s'amuse, ça détend!»

Ce nouveau dialogue ne change pas le sens de la séquence et le téléspectateur n'y perd ni information ni humour.

Toutefois, certaines libertés de traduction ont des conséquences plus importantes car elles ont trait à la mise en scène de l'élément dramatique qui fonde ce numéro de la sitcom.

Dans la cinquième séquence:

- Dans la V.O., Jamie avoue à Paul qu'elle a eu une aventure avant qu'ils emménagent ensemble:

"What you're thinking, I did yes. What you're thinking, I did yes.» Paul réplique: «Esteban? I don't know that song: I think it's Mohammed Hamish.»

- La V.O.S.T. reprend l'idée de la V.O. Jamie dit à Paul: «Oui, ce que tu as en tête, je l'ai fait, oui je l'ai fait.» Et Paul répond, embarrassé: «Demande au groupe. Je ne sais pas si c'est possible. Ce que tu as en tête, je l'ai fait! C'est de qui ?» Paul est gêné par la sortie que vient de faire Jamie devant les autres invités et tente de faire passer les paroles de sa femme pour le titre d'une chanson, l'orchestre se trouvant juste derrière eux.

- En V.F., le traducteur n'a pas du tout gardé ce trait d'humour. Il a modifié la réplique qui n’a plus grand-chose à voir avec sa version originale et qui ne s'inscrit pas très bien dans la séquence: «Avec les bruits de l'orchestre, je sais pas bien ce qu'il en est au juste. C'est... c'est vrai. Ben oui, entre ce qu'on pense et ce qu'on dit, on ne sait pas toujours bien définir la frontière... Excusez-moi, bon appétit.» 
La V.F. est très confuse. Elle occulte l'image d'un Paul inventif et drôle. La réplique est moins lisible pour le téléspectateur. Paul trouve une porte de sortie plutôt plate et sans humour, qui ne compense donc pas l'intensité dramatique liée au fait de la relation entre Jamie et son ex-amant.

Toujours dans la cinquième séquence:

- Dans la V.O., Paul essayant toujours de connaître le secret de sa femme, lui demande si elle a un amant: «Are you having an affair?»

- Dans la V.F., le traducteur fait une faute de temps en traduisant par «T'as eu une aventure?» Ce à quoi Jamie répond que non.

Cette faute de traduction compromet à la fois l'attitude de Jamie et la problématique même de ce numéro de la sitcom. Il y a là une grave négligence de la part du traducteur. Le récit va reposer sur la révélation du secret de Jamie: elle a eu un amant avant d'emménager avec Paul. Dans la V.O., elle réfute avoir actuellement un amant. Elle est fidèle. Il y a un jeu sur la trame temporelle qui donne toute sa subtilité au récit. La V.F. pervertit le sens narratif car Jamie y répond qu'elle n'a pas eu d'amant. Non seulement Jamie est une menteuse, alors que dans le récit original elle dit la vérité, mais, en plus, les téléspectateurs, qui apprendront par la suite qu'elle avait effectivement eu un amant, seront ballottés d'un fait à son contraire, ce qui nuit à la lisibilité de la sitcom. De plus, l'intensité dramatique est modifiée car, dans la V.O., ce qui importe à Paul, c'est de savoir si elle a actuellement un amant. Le fait que cet événement relève du passé va dégonfler son caractère dramatique et va fonder une nouvelle complicité: le couple idéal se dit tout. Dans la V.F., ce qui ennuie Paul, c'est que Jamie ait eu un amant. La révélation de Jamie ne va rien changer à la situation, telle qu'elle est ressentie par Paul. Il n'y aura pas de baisse de l'intensité dramatique.

Dans la sixième séquence, il y a un nouvel écart de traduction lié à la faute précédente:

- Dans la V.O., Jamie explique pourquoi elle a craqué pour cet autre homme, c'est parce qu'ils avaient l'un pour l'autre une attirance: «Stupid little crush.» Elle avoue donc à Paul qu'elle avait un faible pour quelqu'un d'autre.

- En V.O.S.T., la traduction est fidèle: «On était bêtement attirés.»

- En V.F., cette notion d'attirance est complètement occultée car «crush» n’a pas été traduit par attirance mais par "petit accroc», qui n'a rien à voir et n'est en aucun cas un sens possible de «crush». Dans cette version, Jamie n'avoue donc pas avoir eu le béguin pour quelqu'un, mais elle dit que c'était une erreur. Le sens de la phrase est totalement tronqué.

La cause de cet écart par rapport au sens original est à chercher dans l'occurrence précédente. La trame temporelle de la V.O. permet à Jamie de reconnaître une attirance car cette relation avec un amant appartient au passé. Ce qui est en cause d'un point de vue dramatique, c'est que cela n'appartienne pas au présent. Dans la V.F., ce que Paul mettait en cause, c'est une relation passée. Le traducteur est obligé de faire qualifier cette relation d'" erreur » par Jamie pour ressouder le couple et intégrer, sans trop de casse, cet incident conjugal dans l'identité et le positionnement de Dingue de toi. Il y a donc une liberté de traduction qui induit une modification de la trame narrative.

Accessoirement, la traduction est légère car, en V.O. et en V.O.S.T., la phrase est conjuguée au passé alors qu'en V.F., elle est conjuguée au présent: «Il s'agit d'un stupide petit accroc». Ce qui confirme que le traducteur a manqué de vigilance sur la temporalité.

\section{Conclusion}

L'étude comparative de la V.O., de la V.O.S.T. et de la V.F. met en évidence plusieurs types d'artefacts. Nous avons démontré que ces artefacts relèvent soit d'une adaptation à la culture du pays ou de la cible auxquels est destiné le produit audiovisuel, soit d'une modification de l'œuvre en elle-même pour lui donner un autre positionnement, soit d'une transformation de la médiation audiovisuelle ou soit encore d'une altération de la trame narrative.

Ces artefacts sont-ils issus d'une démarche délibérée ou d'un manque de moyens? Pour mettre cette question à l'examen, il peut être utile de comparer la V.O.S.T et la V.F. À l'étude, la V.O.S.T. s'est révélée moins infidèle que la V.F. Certaines références culturelles y sont conservées, la médiation y est contrainte au même équilibre entre les registres verbal et visuel et, enfin, il n'y a pas d'altération de la trame narrative. Le respect relatif de l'œuvre originale dans la V.O.S.T. semble surtout dû à des contraintes d'ordre technique. La V.O.S.T n'est cependant pas exempte d'artefacts. Les personnages s'y expriment de façon vulgaire si nous comparons avec la V.O., ce qui dénote une opération délibérée de repositionnement. Dans la V.F., l'adaptation culturelle devient le prétexte à un changement de cible, pas toujours homogène, ni cohérent du reste. L'œuvre y est considérablement modifiée au travers de la personnalité des personna- 
ges mis en scène. La médiation verbale y est largement renforcée. Par ailleurs, la trame narrative est altérée par des erreurs de traduction. Il y a là de nombreux indices d'une transformation délibérée par la traduction-adaptation. Celle-ci est conçue comme une opportunité de repositionnement de la sitcom, avec l'hypothèse d'une demande différente du public français. Toutefois, les altérations de la trame narrative et les incohérences de reciblage montrent que la traduction-adaptation souffre tout de même d'une insuffisance des moyens consacrés au travail de la post-production.

La traduction-adaptation est conçue pour faciliter l'introduction et la diffusion de la sitcom dans le pays destinataire. Cependant, telle qu'elle est menée, cette traduction-adaptation engendre délibérément des artefacts qui altèrent l'identité originelle de l'œuvre par le lissage, voire la trahison, de ses multiples particularités. Nous sommes donc bien en présence d'un conflit entre les impératifs économiques et la dimension culturelle, liés à un produit audiovisuel. La demande exprimée par une partie du public pour un plus grand maintien de l'originalité des sitcoms ${ }^{1}$ contredit le préjugé, largement partagé par les professionnels et les responsables commerciaux, selon lequel la traduction implique une profonde adaptation. L'idée reçue d'un antagonisme entre l'authenticité culturelle d'un produit et les exigences commerciales mériterait un réexamen. La multiplication des chaînes accessibles, notamment étrangères, et la progression des échanges culturels conduisent le public à évoluer. Il se pourrait que le public soit en mesure d'apprécier les produits audiovisuels dans toute leur originalité. Quoi qu'il en soit, la politique menée par les commanditaires et les professionnels de la traduction-adaptation des produits audiovisuels s'inscrit dans une vision réductrice, voire régressive, du public.

YANNICKE LEBTAHI

Université de Lille 3, Lille, France yannicke.lebtahi@wanadoo.fr

\title{
NOTES
}

1. Suite au succès de la diffusion en V.O.S.T. de Friends sur Canal Jimmy, le public français a pris connaissance de la possibilité de recevoir une version plus originale. France 2 ayant acquis les droits, elle se contenta d'une diffusion exclusive en V.F. Les réclamations indignées du public la conduisent après bien des atermoiements à programmer enfin la V.O.S.T. en fin de soirée.

\section{RÉFÉRENCE}

Jost, F. (1999): Introduction à l’analyse de la télévision, Paris, Ellipses.

\section{Aptitude to Interpreting: Preliminary Results of a Testing Methodology Based on Paraphrase}

\author{
RÉSUMÉ \\ Cet article présente les premiers résultats d'une étude longitudinale appliquée à un échantillon de 46 étudiants \\ de la SSLMIT de Trieste. L'objectif de l'étude est la mise au point d'un test d'aptitude pour les candidats aux \\ cours universitaires d'interprétation de conférence. Le test consiste en un exercice de paraphrase en simultanée. \\ L'analyse linguistique des prestations selon trois niveaux, syntaxique, sémantique et pragmatique, a permis \\ d'établir des corrélations, significatives au point de vue statistique, entre les résultats du test, les notes aux \\ examens et le temps employé par les étudiants pour conclure leur formation en interprétation.
}

This article presents the first results of a longitudinal study involving a sample of 46 students of the SSLMIT of Trieste. The study aims at developing an aptitude test for candidates to Conference Interpreting courses at university level. The test is based on an exercise of simultaneous paraphrasing. The linguistic analysis of the performances at syntactic, semantic and pragmatic levels has shown statistically significant correlations between the test results, the marks of interpreting exams and the time spent by the students to complete their training in interpreting.
\end{abstract}

MOTS-CLÉS/KEYWORDS

predictivity, coherence, syntax transformation, synonym, deletion

\section{Introduction}

To what extent conference interpreters are a product of nature or nurture is still a moot point in the interpreting community. However, the need for candidates to harbour legitimate academic and professional 